\title{
A PÓS GRADUAÇÃO EM EDUCAÇÃO NO BRASIL E O PLANO NACIONAL DE PÓS- GRADUAÇÃO - PNPG - 2005-2010: LEITURAS SOBRE O MODELO DE DESENVOLVIMENTO, AVALIAÇÃO, SUSTENTABILIDADE E IMPACTO LOCAL.
}

Luis Enrique Aguilar ${ }^{1}$

\section{Introdução}

Este "paper" foi construído com o objetivo de somar uma perspectiva às discussões que são realizadas no Brasil com motivo da consulta que o Ministério de Educação e Ciência (MEC) vem fazendo às instituições e associações científicas brasileiras sobre o V - Plano Nacional de PósGraduação(V-PNPG). Nosso desafio no contexto do Projeto ALFA é trazer leituras do modelo de desenvolvimento, sustentabilidade e impacto local do ensino superior no país estabelecendo interfaces com o papel que, nesta conjuntura, lhe cabe à sub-área de educação.

Retomamos para tanto subsídio apresentados pela Associação Nacional de PósGraduação e Pesquisa em Educação (ANPEd) ${ }^{2}$ em meados de 2004, quando o processo de consulta oficial do MEC tinha sido deflagrado. Neste documento a ANPED expressa sua preocupação com "o desenvolvimento e à consolidação do ensino de pós-graduação e da pesquisa na área de Educação no Brasil e compreende que a Educação, de modo geral, e, especificamente, a do ensino superior e a de estudos pós-graduados, devem ser objeto de atenção privilegiada do governo, da comunidade científica e da sociedade como um todo". Reconhece também "a importância de políticas eficientes e duradouras, capazes de impulsionar o desenvolvimento científico e tecnológico do país".

Neste sentido a ANPEd entende a diversidade dos pressupostos que sustentam as questões pertinentes ao campo da Pós-Graduação em Educação e cuja presença considera indispensável para a formulação e implementação de uma política nacional de pós-graduação. Assim enfatiza: a estrita vinculação e intima articulação da pós-graduação em educação com o sistema nacional de educação; o caráter imprescindível das políticas de Estado para o ensino e para a pesquisa; a institucionalização da pesquisa com caráter humanitário e social; a defesa do mestrado

\footnotetext{
1 Pesquisador do Laboratório de Políticas Públicas e Planejamento Educacional e Prof. do Programa de Pós Graduação em Educação em Educação da Faculdade de Educação da UNICAMP 2 Ver Documento ANPED, - Subsídios da Associação Nacional de Pós Graduação e Pesquisa em Educação ao V Plano Nacional de Pós-Graduação, Caxambu, Novembro de 2004 ANPEd -Associação civil, sem fins lucrativos, fundada em 1978, constitui-se de sócios institucionais (Programas de Pós-Graduação em Educação, atualmente em número de 66) e de sócios individuais (professores, pesquisadores, mestrandos e doutorandos e demais pesquisadores da área). Abriga, em sua estrutura, 21 Grupos de Trabalho (GT) e 2 Grupos de Estudos (GE), uma estrutura que lhe dá sustentação e suporte acadêmico e científico.
} 
acadêmico, ancorada no significado pleno da formação de pesquisadores em educação resguarda sua especificidade; a sustentabilidade do sistema de pós graduação com suporte na eficácia da avaliação e das relações de cooperação interinstitucional; a reformulação da matriz orçamentária que encerre o déficit de docentes e pesquisadores; a necessidade de uma política de formação e qualificação de quadros; interromper a degradação da infra-estrutura de pesquisa nas universidades públicas do país, crie um sistema de bolsas mais abrangente e concretiza investimentos mais efetivos;

\section{Cenários da implementação do V-PNPG e a Pós -Graduação em Educação brasileira.}

A reflexão inicial que queremos apresentar tem o predomínio da análise contextual que desenha o cenário da implantação do V-PNPG e que no nosso modo de ver, requer uma profunda reflexão pautada pelo ponto de partida da análise que remete à história da constituição da pós-graduação em educação. Este fato lhe outorgará especial significado à análise da apertura e encerramento de ciclos de desenvolvimento da pós-graduação em educação brasileira. Trata-se por tanto de localizar esse ponto de partida que faz com que, até 2005, tenhamos percorrido, na área de educação, aproximadamente quatro(4) décadas desde que, pela primeira vez, foi reconhecida um atividade precursora da pós-graduação em educação, quando se abria um ciclo de desenvolvimento e formação de quadros que daria ao país o cenário da pós-graduação em educação que hoje ostenta.

A conjuntura atual recoloca este desafio de desenvolvimento e formação de quadros que, efetivamente possui outra lógica e outras influências, após quarenta anos. Constata-se que há uma intensa renovação de quadros que, afetada por novas variáveis como o impacto de reformas nas leis previdenciárias brasileiras, (re) configura sua trajetória. É possível, identificar o início de um novo ciclo de desenvolvimento da pós-graduação em educação, pela aposentadoria da maioria dos fundadores da pós-graduação em educação e/ou pela aposentadoria e migração de instituições públicas para as instituições privadas, que deverá re-pensar a trajetória construída e imaginar o futuro a partir deste cenário de renovação.

Neste contexto e analisando o Documento do V-PNPG destacamos o seu reconhecimento "da educação como fator estratégico do processo de desenvolvimento sócio-econômico e cultural do país como princípio”. Compreendemos que não se trata de um reconhecimento da sub-área de educação e sim dos processos formativos educacionais que estão implícitos na palavra educação de modo geral. Por isto resgatamos duas idéias centrais que dimensionam a responsabilidade atribuída à educação e que estão presentes na política que sustenta o V-PNPG: a) a concepção de sistema (sistema de pós-graduação) -o que de fato impõe conceitualmente re-leituras das inter-relações no 
interior do universo da pós-graduação no país, e b)a definição, embora quase-implícita, dos contornos de uma responsabilidade estratégica no desenvolvimento que vincula os afazeres da pósgraduação em educação ao sistema educacional brasileiro. Observe-se uma mudança e re-difinição do foco da política e os possíveis impactos desta indução que reduz potencialmente a abrangência do significado da pesquisa em educação.

Um outro aspecto está referido às políticas de expansão do sistema nacional de pós-graduação que podem ser analisados a partir de leituras indutivas da qualificação do ensino superior brasileiro e de leituras das modalidades que assumiu a institucionalização da pesquisa, duas premissa permanentes de todos os 4(quatro) PNPGs anteriores .

Recuperando este histórico dos PNPGs, foi a constatação de demandas sobre as universidades e instituições de pesquisa, no sentido de "formar, em volume e diversificação, pesquisadores, docentes e profissionais e encaminhar e executar projetos de pesquisa, assessorando o sistema produtivo e o setor público" (MEC, 1975:12), a que fez com que o I Plano Nacional de PósGraduação definira que caberia ao MEC o atendimento da primeira demanda, pois a responsabilidade no atendimento da segunda seria compartilhada com outros órgãos governamentais. Institucionalizar isto requereria de programas de capacitação.

Já no II Plano Nacional de Pós Graduação (1982-1985), o objetivo central continua a ser a formação de recursos humanos qualificados para as atividades docentes, de pesquisa e técnicas visando o atendimento dos setores público e privado.

A questão central do II PNPG não foi apenas a expansão da capacitação docente, mas a elevação da sua qualidade, enfatizando-se, nesse processo, a importância da avaliação, da participação da comunidade científica e do desenvolvimento da pesquisa científica e tecnológica.

A partir do III Plano Nacional de Pós-Graduação (1986-1989), com mais clareza se expressa a conquista da autonomia nacional, no entanto não há um quantitativo de cientistas suficiente para se atingir plena capacitação científica e tecnológica. Deve haver um processo da formação de recursos humanos de alto nível, considerando que a sociedade e o governo pretendem a independência econômica, científica e tecnológica para o Brasil, no próximo século. A ênfase principal desse plano está no desenvolvimento da pesquisa pela universidade e a integração da pósgraduação ao sistema de ciência e tecnologia. O plano acrescenta a necessidade de institucionalização e ampliação das atividades de pesquisa como elemento indissociável da pósgraduação e de sua integração ao sistema nacional de ciência e tecnologia. Estabelece a universidade como ambiente privilegiado para a produção de conhecimento, enfatizando-se o seu papel no desenvolvimento nacional. 
Os objetivos desse plano foram:

- A consolidação e a melhoria do desempenho dos cursos de pós-graduação;

- A institucionalização da pesquisa nas universidades para assegurar o funcionamento da pósgraduação;

- A integração da pós-graduação ao setor produtivo.

O III PNPG, além das diretrizes e recomendações gerais para a pós-graduação e pesquisa, traz medidas específicas para a institucionalização da pesquisa, tais como destacar, nos orçamentos das universidades, verbas específicas para a pesquisa e a pós-graduação, reestruturar a carreira docente a fim de valorizar a produção científica tanto para o ingresso como para a promoção, planejar e ampliar os quadros universitários e institucionalizar a atividade sabática e o fortalecimento do pósdoutorado, além de efetuar a atualização das bibliotecas e das informações científicas e de laboratórios.

Os PNPGs até o presente produziram:

- Integração da pós-graduação no interior do sistema universitário, institucionalizando a atividade de pesquisa;

- Construção de um amplo (embora insuficiente) sistema de bolsas no país e no exterior, que tem contribuído para a qualificação e reprodução do corpo docente e de pesquisadores;

- Estruturação de uma política de apoio financeiro (mais abrangente) aos programas de pósgraduação;

- Implantação de um sistema nacional de avaliação (menos homogeneizante) dos programas, realizado por meio de julgamento de pares;

- Integração do ensino à pesquisa, estabelecendo-se um número limitado de disciplinas articuladas com as respectivas linhas de pesquisa dos cursos;

- Fortalecimento da iniciação científica;

- Criação de um eficiente sistema de orientação de dissertações e de teses;

- Articulação da comunidade acadêmica nacional com relevantes centros da produção científica internacional.

- O desenvolvimento do país esteve sempre atrelado ao desenvolvimento da pós- graduação.

Este desenvolvimento entendido : 
- como Formação de pesquisadores e

- como produção e sistematização de conhecimento através da pesquisa.

- como espaço privilegiado para que as Atividades de Ensino estejam ancoradas em Propostas Curriculares coerentes e Atividades de Pesquisa capazes de produzir e reproduzir conhecimento.

A integração do ensino à pesquisa ocorre nesta dinâmica do desenvolvimento institucional. Daí que as expressões que denominamos cultura de docência estão ligadas às leituras da evolução histórica da pós-graduação e é possível afirmar que esta cultura como forma ou etapa evolutiva das tradições e valores institucionais esteja no início das atividades e ritos de ensinar nas universidades.

Nesta perspectiva e particularmente nas públicas podemos reconhecer que a integração do ensino à pesquisa foi o ponto essencial capaz de criar uma nova tradição e novos valores institucionais dando passo à Cultura de pesquisa. A produção de conhecimento começou e não se deteve mais.

O desenvolvimento da Pós-graduação e especialmente sua expansão nas últimas três décadas tem sido um produto conjuntural e espontâneo e somente a partir das políticas de regulação das duas últimas gestões (Fernando Henrique Cardoso 1994-2002 e Luiz Inácio Lula da Silva 2002-2005) é possível reconhecer a indução de um redesenho da relação público-privado pelo crescimento exponencial desta última dimensão $o^{3}$.

Em paralelo a este fator relevante do crescimento quantitativo corre a demanda de qualificação no sistema de pós-graduação e esta (a demanda) uma clara amostra da relação público-privado, onde os programa de pós-graduação da universidade pública tiveram e têm um papel fundamental neste desenvolvimento. Há uma outra faze da qualificação do ensino superior que ocorre como um subproduto das políticas previdenciárias que induziram a migração de quadros da universidade pública para a universidade privada provocando benefícios no sistema todo especialmente criando as bases de culturas pesquisa antes privilégio das universidades públicas.

As políticas de desenvolvimento sustentável do Sistema de pós-graduação ancorado em estratégias de regionalização, cooperação nacional e internacional de modo a superar os ciclos de desenvolvimento que se encerram e abrem nas instituições universitárias públicas e privadas brasileiras. As políticas que induzem a cooperação nacional poderiam efetivamente ser um fator equilibrador do desenvolvimento nacional da pós-graduação, criando estratégias regionais diferenciadoras de incentivo. Os programas atuais, também comparados com a demanda requerem

3 SINAES - Sistema Nacional de Avaliação da Educação Superior -Censo Nacional da Educação Superior -Resumo técnico - 2003, INEP,MEC,Brasília,2004 
de uma redefinição dos investimentos procurando atingir universos maiores nas regiões com baixo desenvolvimento.

Vejamos a Evolução dos Cursos visando qualificação de quadros por Região Administrativa Mestrado e Doutorado - Distribuição Desigual

\begin{tabular}{|c|r|r|r|r|}
\hline & \multicolumn{3}{|c|}{ Mestrado } & \multicolumn{2}{|c|}{ Doutorado } \\
\cline { 2 - 3 } & 1996 & 2004 & 1996 & 2004 \\
\hline Região & 685 & 1076 & 450 & 689 \\
\hline Sudeste & 166 & 384 & 50 & 177 \\
\hline Sur & 155 & 305 & 22 & 107 \\
\hline Nordeste & 53 & 126 & 12 & 42 \\
\hline Centro-Oeste & 24 & 68 & 7 & 19 \\
\hline Norte & & & & \\
\hline
\end{tabular}

Fonte: CAPES/MEC (V-PNPG 2005-2010- Documento)

Se analisamos uma outra vertente do cenário do desenvolvimento, da sustentabilidade e do impacto local a partir da relação PIB, número de docentes e valor dos investimentos efetuados em bolsas pelas agências do fomento, pode observar-se a assimetria apontada inicialmente, em contraste com uma alta concentração onde existe maior capacidade instalada de recursos humanos e infra-estrutura

"O crescimento de grupos emergentes é prejudicado numa situação de orçamentos reais decrescentes e da tendência de políticas públicas de canalizar parte significativa dos investimentos nos grupos mais consolidados. O mesmo ocorre em novas áreas do conhecimento e naquelas já consolidadas que se encontram localizadas em regiões de menor concentração de massa crítica”. (V-PNPG 2005-2010)

O diagnóstico dessa situação aponta para a necessidade da formulação de estratégias específicas visando à criação de novos paradigmas para a evolução do sistema, atendendo as prioridades nacionais. Caso contrário, nos próximos anos se observará a continuidade do crescimento da pós-graduação e permanência das assimetrias regionais.

Relações entre \% Docentes e \% PIB e entre

$\%$ Investimentos em bolsas e \% Docentes

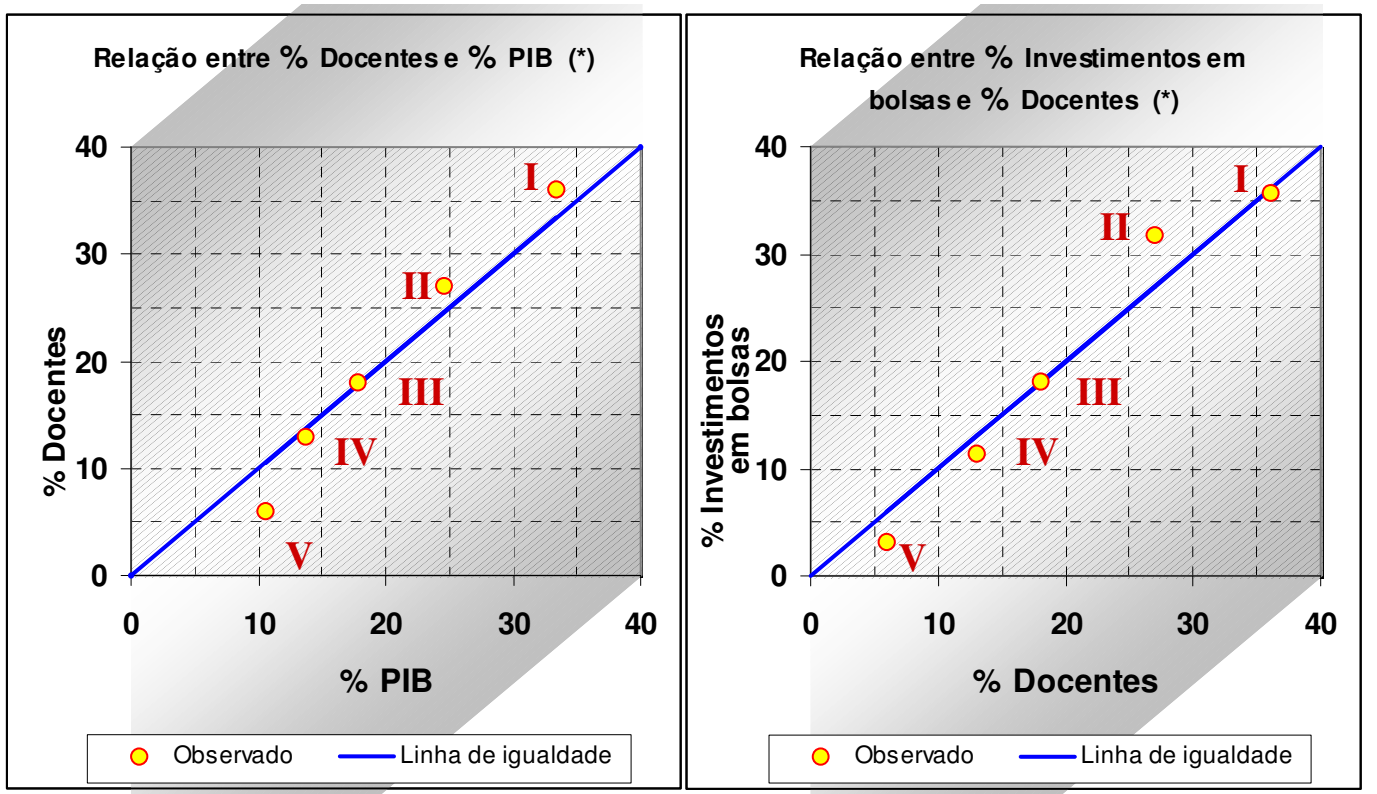

Cada ponto no gráfico representa uma área geográfica, conforme a seguinte legenda: 
Apresentamos para debate, um dos dilemas da articulação entre "bases ou ênfases" do modelo desenvolvimento da pós-graduação, possibilidades de análise da sustentabilidade e impacto local, correlacionando, assimetrias regionais e prioridaes do V-PNPG. Como o PNPG aponta, “ não se pode pensar em reduzir os investimentos nos grupos mais qualificados. Ao mesmo tempo, torna-se necessário criar condições adequadas para o desenvolvimento dos grupos já estabelecidos em regiões com menor densidade de grupos de pesquisa ou em áreas do conhecimento estratégicas para o desenvolvimento harmônico da ciência e tecnologia nacional. Isso implica no estabelecimento de propostas indutivas que contemplem recursos novos elou remanejamento de orçamentos. As iniciativas para correção da tendência deveriam começar pelo reconhecimento, por parte dos governos estaduais, da importância da qualificação de recursos humanos locais para propiciar o desenvolvimento do estado e da região. O ponto de partida seria a absorção de doutores em áreas específicas de modo a formar massa crítica capaz de propor programas de pós-graduação de qualidade.

O crescimento verificado nos últimos anos no Sistema Nacional de Pós-Graduação caracteriza-se como um marco histórico para o desenvolvimento científico e tecnológico, ancorado por políticas consistentes da pós-graduação brasileira. Todavia, há necessidade de se propor um novo modelo de crescimento para os anos vindouros, incorporando modificações conceituais e organizacionais que atenuem as desigualdades regionais, intra-regionais e entre estados, bem como, as assimetrias entre áreas do conhecimento".(V-PNPG- 2005-2010)

As discussões de futuro da pós-graduação e suas implicações à luz das políticas de avaliação e financiamento permitam retomar o elo que articula, o papel da pós-graduação em educação, sua trajetória, e seu local no contexto do V-PNPG 2005-2010, pois o debate travado é crucial para seu destino e para as expectativas que o próprio plano depositou na sub-área de educação e a posição do conjunto da sub-área cujos argumentos vão além desta expectativa criando um cenário de conflito em visão prospectiva porque se retomam os dilemas próprios de sua especificidade. 
Num cenário de intensa renovação, resulta imprescindível, retomar a estrita vinculação e intima articulação da pós-graduação em educação com o sistema nacional de educação em oposição a aceitar um papel reducionista para a educação no contexto do desenvolvimento nacional; o caráter imprescindível das políticas de Estado para o ensino e para a pesquisa; a institucionalização da pesquisa com caráter humanitário e social; a defesa do mestrado acadêmico, ancorada no significado pleno da formação de pesquisadores em educação resguarda sua especificidade; a sustentabilidade do sistema de pós-graduação com suporte na eficácia da avaliação e das relações de cooperação interinstitucional; a reformulação da matriz orçamentária que encerre o déficit de docentes e pesquisadores; a necessidade de uma política de formação e qualificação de quadros; interromper a degradação da infra-estrutura de pesquisa nas universidades públicas do país, crie um sistema de bolsas mais abrangente e concretiza investimentos mais efetivos.

\section{Referências Bibliográficas}

ANPED - Associação Nacional de Pós Graduação e Pesquisa em Educação, Subsídios ao Plano Nacional de Pós-Graduação, Caxambu, Novembro de 2004.

Comissão de Elaboração do Plano Nacional de Pós-Graduação, Portarias CAPES Nº:046/ 2004 de 19 de maio de 2004; No:053/ 2004 de 22 de junho de 2004 e $\mathrm{N}^{\mathrm{o}}: 083 / 2004$ de 29 de setembro de 2004, Brasília, DF, 2004.

Comissão Interministerial para o Desenvolvimento da Pós-Graduação e da Ciência e Tecnologia Relatório Final (Sumário Executivo) CAPES/CNPq Portaria Interministerial MCT/MEC N ${ }^{\circ}$ 0270 de 22 de maio de 2003, Brasília, DF, 2003

Plano Nacional de Pós-Graduação (PNPG) 2005-2010 Ministério da Educação MEC Coordenação de Aperfeiçoamento de Pessoal de Nível Superior - CAPES - Brasília, DF, dezembro de 2004

SINAIS - Sistema Nacional de Avaliação da Educação Superior - Censo da Educação Superior 2003 Resumo Técnico, Ministério da Educação e Ciência - Instituto Nacional de Estudos e Pesquisas Educacionais, Brasília, DF, 2004. 